\title{
An Apple Watch a day keeps the doctor away?
}

\author{
Mikołaj Basza ${ }^{1 *}$, Bartosz Krzowski ${ }^{2 *}$, Paweł Balsam², \\ Marcin Grabowski ${ }^{2}$, Grzegorz Opolski ${ }^{2}$, Łukasz Kołtowski ${ }^{2}$ \\ ${ }^{1}$ Medical University of Silesia in Katowice, Poland \\ ${ }^{2} 1^{\text {st }}$ Department of Cardiology, Medical University of Warsaw, Poland
}
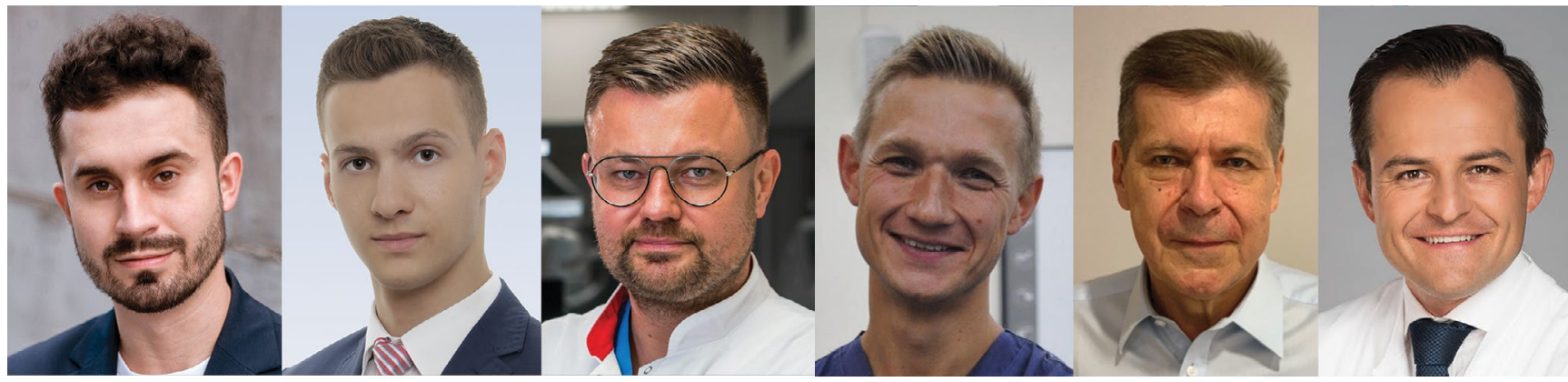

The high prevalence of cardiovascular diseases and an increasing health workforce shortage are major challenges that cardiology will face in the decades to come. New technologies supporting physicians in screening and patient monitoring are considered a potential solution to help fill this gap. For example, the average smartwatch user generates millions of basic vital sign data points each day, observed almost continuously 7 days a week in a patient's natural environment. 2019 ended with more than 500 million wearable users, and according to the market forecasts, this number will have reached over 1 billion by 2022 [1]. Apple dominates the smartwatch market with Apple Watch (AW), which is recognized as a Food and Drug Asscoiation class-2 medical device; nevertheless, its applications and reliability in medicine remain unclear.

The AW collects various biosignals based on two core technologies, photoplethysmography (PPG) and single-lead electrocardiography (ECG) [2].
Using green or infrared light, PPG is used to measure heart rate (HR) and HR variability, relying on blood volume changes in tissue. According to manufacturer information, the AW supports an HR range of 30-210 bpm. Measurements of HR are taken in the background during rest, but a user can also perform it on-demand. In many studies with similar conclusions, HR measurement accuracy has been validated on a healthy population during different activities. Overall accuracy during rest, low and moderate activity is comparable to standard 12-lead ECG and decreases near range thresholds with motion artifacts [3-5]. These findings suggest a possible use of AW HR monitoring during therapy and cardiac rehabilitation. Apple offers an HR notification function that informs the user when the HR in a 10 min resting period is out of the previously set range. The irregular rhythm notification function is widely described in the Apple Heart Study [6]. AW showed a $34 \%$ diagnostic yield in detecting

Address for correspondence: Łukasz Kołtowski, MD, PhD, FESC, $1^{\text {st }}$ Chair and Department of Cardiology, Medical University of Warsaw, ul. Banacha 1a, 02-097 Warszawa, Poland, tel: +48 2259929 58, fax: +48 22 599-19-57, e-mail: lukasz@koltowski.com

Received: 29.10.2020 Accepted: 5.05.2021

*Equal contribution

This article is available in open access under Creative Common Attribution-Non-Commercial-No Derivatives 4.0 International (CC BY-NC-ND 4.0) license, allowing to download articles and share them with others as long as they credit the authors and the publisher, but without permission to change them in any way or use them commercially. 


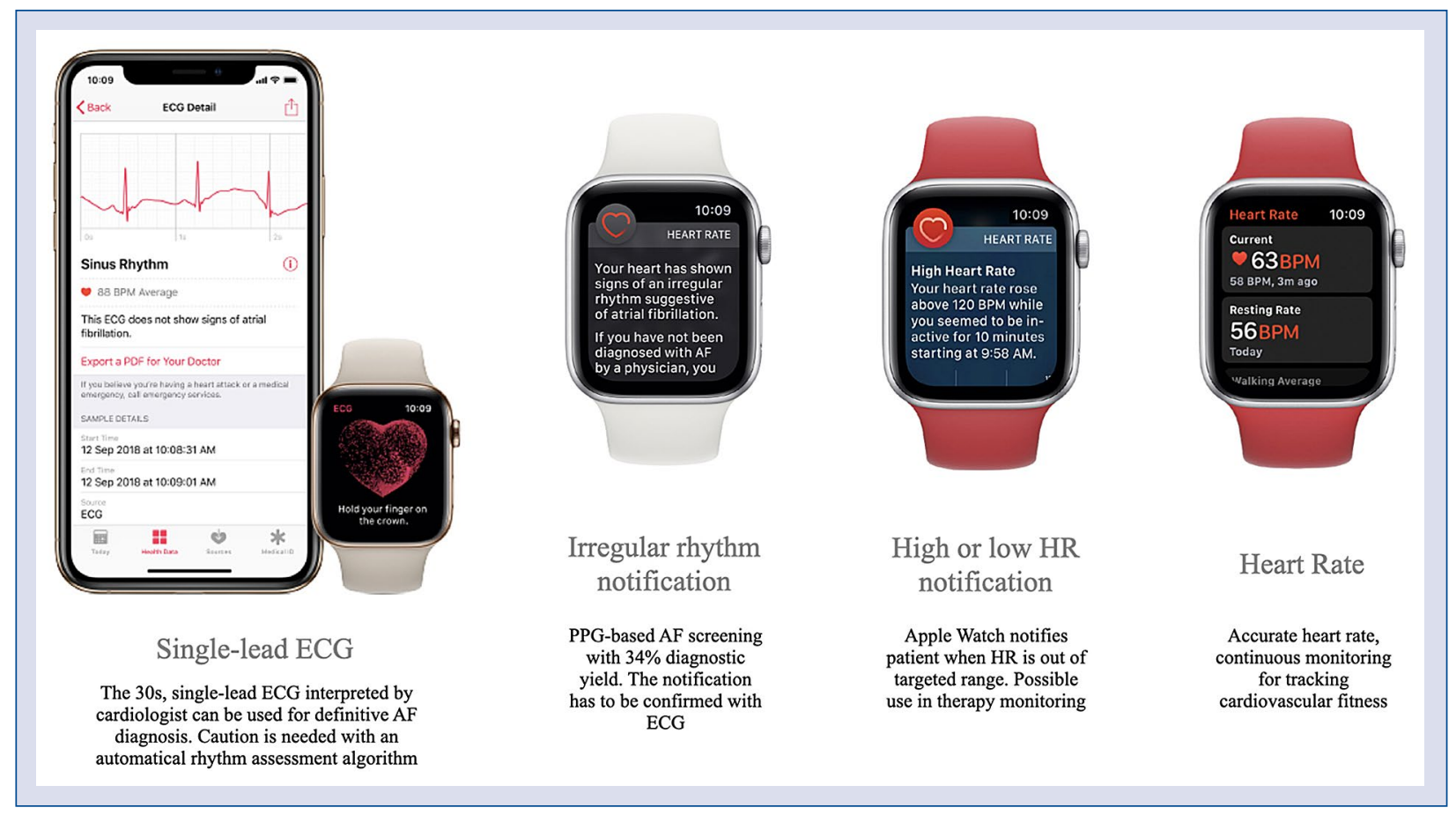

Figure 1. Main Apple Watch options are available for everyday use; AF — atrial fibrillation; HR — heart rate; ECG — electrocardiogram; PPG — photoplethysmography.

atrial fibrillation $(\mathrm{AF}), 35 \%$ in the population over 65 years old and $18 \%$ in the cohort younger than 40 years of age. $89 \%$ of true positives had AF episodes lasting at least 1 hour. The positive predictive value of individual notification was $84 \%$. The high false-positive rate was the main allegation in this study. However, ECG patches used for validation were worn for an average of 6.3 days and a few days after initial notification and by a limited number of study participants, so the absence of AF during validation does not indicate that the notification was false. Especially in paroxysmal and infrequent episodes of arrhythmia. This study's findings imply the potential benefits of irregular pulse notification in $\mathrm{AF}$ screening in a population over 65 years old when AF screening is cost-effective. According to the European Society of Cardiology (ESC) 2020 guidelines, methods based on PPG require additional ECG confirmation, which is possible in Apple Watch Series 4 and higher and occasionally, in case of doubt, needs to be verified with traditional 12-lead ECG tracing (Fig. 1).

The Apple Watch Series 4 and higher have built-in two electrodes, a titanium electrode in the Digital Crown, and a chromium silicon carbon nitride layer applied on the back of the AW. They allow obtaining a single lead ECG corresponding to lead I in standard ECG. In a validation study,
Apple showed high morphology agreement between Apple Watch ECG and 12-lead ECG (lead I) waveforms at rest and after exercise. Sub-study of the Leipzig Apple Heart Rhythm Study compared three leads obtained using alternative positioning of Apple Watch to leads I, II, III of standard 12lead ECG with the correlation between $97 \%$ and 99\% for a duration (ms) of $\mathrm{P}$ wave, $\mathrm{PR}$ interval, QRS complex, QT interval and $\mathrm{T}$ wave in all three leads. The polarity concordance of $\mathrm{P}$ wave, QRS complex, and $\mathrm{T}$ wave was $98-100 \%$. However, a lower correlation - 72-90\% - has been observed for those parameters' amplitude (mV). In 2020 Spaccarotella et al. [7] obtained leads I, II, III, $\mathrm{V}_{1}$, $\mathrm{V}_{2}, \mathrm{~V}_{3}, \mathrm{~V}_{4}, \mathrm{~V}_{5}$, and $\mathrm{V}_{6}$ using AW and compared it to 12-lead ECG on 100 patients admitted to the coronary care unit. Bland-Altmann did not show a significant difference in millimeters of ST-segment deviation. The feasibility of AW ECG interpretation in $\mathrm{AF}$ detection has been confirmed with 96\% sensitivity and $100 \%$ specificity [8]. Those studies have shown that Apple Watch ECG waveform is a clinically relevant biosignal and meets the criteria of ESC 2020 guidelines for AF definitive diagnosis, which is especially important in patients at high risk of stroke. Single lead ECG is the basis of the Apple Watch ECG app 1.0 algorithm that, according to the manufacturer, is characterized by 
98.3\% sensitivity and $99.6 \%$ specificity in detecting AF. Still, this data requires cautious interpretation. An independent study conducted on 50 patients, who had undergone cardiac surgery, monitored by telemetry, showed $41 \%$ specificity in detecting $\mathrm{AF}$ by an algorithm and 5 times higher unreadable ECGs rate [8]. Apple Watch ECG app 2.0 algorithm interprets the rhythm automatically and classifies it into sinus rhythm, AF, low or high heart rate, inconclusive and poor recording. Apple's internal study demonstrated $98 \%$ correctly classified sinus rhythms and AF in the HR range of 50-99 bpm and $93 \%$ for sinus rhythm, and $83 \%$ for AF in HR range 100-150 bpm. There are no other studies that confirmed the results of the ECG 2.0 Clinical Validation Study.

One of the AW ECG waveform's possible applications is screening and monitoring therapy with a cardiac adverse effect. In 2020 Strik et al. [9] validated $\mathrm{QT}$ interval measurements using AW ECG to assess potential usefulness in patients with COVID-19 on hydroxychloroquine/azithromycin treatment. He revealed that adequate QT measurements were possible from AW ECG in $85 \%$ of patients. However, using additionally lead II and $\mathrm{V}_{6}$ obtained using AW increased that score to $94 \%$.

Year by year, the manufacturers of wearables are getting deeper into healthcare by providing new medical features and conducting further studies in collaboration with leading medical institutions. In Apple Watch Series 6, optical sensors were redesigned to provide oxygen saturation and $\mathrm{VO}_{2}$ max measurements. Although the blood oxygen level app is officially not intended for medical use, it might be a medical-grade feature in the future. Apple recently has launched two new studies investigating the feasibility of the AW in cardiology. One is related to monitoring heart failure patients, and the second is focusing on the correlation between physical activity and heart health [10].

Though there is a need for further validation, studies have shown that wearables such as AW should not be viewed just like gadgets. Still, they may become a reliable source of medical data. In addition, professional guidance and clinical experience could provide enormous potential in screening arrhythmias, supporting therapy, and cardiac rehabilitation.

Conflict of interest: None declared

\section{References}

1. Thomas Barnett J, Shruti Jain, Usha Andra, Taru Khurana. Cisco Visual Networking Index (VNI) Global and Asia Pacific Mobile Data Traffic Forecast, 2017-2022. 2019.

2. Apple Inc. Using Apple watch for arrhythmia detection. 2020.

3. Khushhal A, Nichols S, Evans W, et al. Validity and reliability of the Apple Watch for measuring heart rate during exercise. Sports Med Int Open. 2017; 1(6): E206-E211, doi: 10.1055/s-0043120195, indexed in Pubmed: 30539109.

4. Falter M, Budts W, Goetschalckx K, et al. Accuracy of Apple Watch measurements for heart rate and energy expenditure in patients with cardiovascular disease: cross-sectional study. JMIR Mhealth Uhealth. 2019; 7(3): e11889, doi: 10.2196/11889, indexed in Pubmed: 30888332.

5. Nelson BW, Allen NB. Accuracy of consumer wearable heart rate measurement during an ecologically valid 24-hour period: intraindividual validation study. JMIR Mhealth Uhealth. 2019; 7(3): e10828, doi: 10.2196/10828, indexed in Pubmed: 30855232.

6. Perez MV, Mahaffey KW, Hedlin H, et al. Large-Scale assessment of a smartwatch to identify atrial fibrillation. N Engl J Med. 2019; 381(20): 1909-1917, doi: 10.1056/NEJMoa1901183, indexed in Pubmed: 31722151.

7. Spaccarotella CA, Polimeni A, Migliarino S, et al. Multichannel electrocardiograms obtained by a smartwatch for the diagnosis of ST-segment changes. JAMA Cardiol. 2020; 5(10): 1176-1180, doi: 10.1001/jamacardio.2020.3994, indexed in Pubmed: 32865545.

8. Seshadri DR, Bittel B, Browsky D, et al. Accuracy of Apple Watch for detection of atrial fibrillation. Circulation. 2020; 141(8): 702-703, doi: 10.1161/CIRCULATIONAHA.119.044126, indexed in Pubmed: 32091929.

9. Strik M, Caillol T, Ramirez FD, et al. Validating qt-interval measurement using the Apple Watch ECG to enable remote monitoring during the COVID-19 pandemic. Circulation. 2020; 142(4): 416-418, doi: 10.1161/CIRCULATIONAHA.120.048253, indexed in Pubmed: 32478565.

10. MacRae CA. Apple Heart \& Movement Study (NCT04198194). 2019 . 